\title{
Advanced biomedical multiphoton fluorescence microscopy with a large band excitation system
}

\author{
Thomas Hortholary ${ }^{\mathrm{a}, \mathrm{b}}$, Claire Carrion ${ }^{\mathrm{c}}$, Claire Lefort*a \\ ${ }^{a}$ CNRS UMR 7252, XLIM Research Institute, Université de Limoges, France \\ ${ }^{b}$ ENS Cachan, 61 avenue du Président Wilson, Cachan, France \\ ${ }^{c}$ BISCEm, Microscopy core Facility Université de Limoges, France
}

\begin{abstract}
Commercial multiphoton microscopes currently include standard and bulk titanium-sapphire laser sources (Ti: Sa) delivering a narrow spectral bandwidth of $10 \mathrm{~nm}$ at the full-width at half maximum. Such a spectral width precludes the instantaneous imaging of more than four fluorophores simultaneously in optimal conditions of target. Femtosecond ultrawideband laser system (UWLS) appeared since fifteen years with an approach of spectral broadening of Ti: Sa pulses into a photonic crystal fiber associated with a dispersion compensation system. In the current work, we demonstrate the interest of an alternative laser solution, compact, cheap, simple and turn-key based on a UWLS delivering a unique spectral excitation bandwidth named supercontinuum. We have coupled this system to a passive filtering setup. Thanks to this unique spectral bandwidth, eleven fluorophores are imaged with multiphoton processes without any modification on the excitation parameters.
\end{abstract}

Keywords: multiphoton microscopy, living sample imaging, supercontinuum laser excitation

*claire.lefort@xlim.fr

\section{INTRODUCTION}

The invention of microscope during the $17^{\text {th }}$ century has played a key role in many domains of life sciences: fundamental biological researches, plant studies and medical discoveries. Since the middle of the $20^{\text {th }}$ century, the widefield microscope was upgraded with the use of the fluorescence light coupled with dyes or antibodies specifically targeting subcellular compartments or molecular components [1]. Optical microscopy was again upgraded since the 90's with confocal microscopy delivering a better resolution with a 3D imaging ability and became the gold standard in biomedical microscopy [2]. It allowed a better knowledge of the cell from inside with the simultaneous observation of several targets. If fluorescence and confocal microscopy have extensively used exogenous dyes labeled with fluorochromes, many fields of life science explore the endogenous fluorescence of cells and tissue in order to find a specific signature of a particular cellular state or the presence of a disorder. Moreover, some samples from life sciences are highly fluorescent by themselves, in particular plants for which the use of exogenous fluorochromes looks not adapted [3]. Finally, these linear methods of microscopy are still limited by the UV-visible excitation range cumulating a restricted acquisition depth with a risk of phototoxicity.

Multiphoton microscopy (MPM) appeared in the 90's, synchronous with the need in improving microscopy methods for life sciences imaging. This nonlinear microscopy method has brought new opportunities in 3D and multichannel imaging, arousing a scale change in the deciphering of the mechanisms implied in the cellular biology. Actually, MPM presents some physical performance unequaled by widefield or confocal microscopy [2, 4-6]. In particular, the excitation 
wavelengths in the near infrared range (NIR) reduce the risks of phototoxicity and can penetrate deeper inside explanted organ or even in vivo. Despite this great interest of MPM in life sciences, a technological breakthrough is still mandatory to make this method the new gold standard in biomedical microscopy. Indeed, in a routine use, MPM is spectrally limited by the excitation laser usually adopted in commercial multiphoton systems: a mode-locked titanium-doped sapphire laser (Ti: Sa, $150 \mathrm{fs}, 80 \mathrm{MHz}, 1 \mathrm{~W}$ ) having a spectral bandwidth of $10 \mathrm{~nm}$, tunable on the range 650 and 1100 $\mathrm{nm}$. With such a bandwidth, only two multiphoton probing processes can be accessible simultaneously: two-photon fluorescence (TPF) and second harmonic generation (SHG). By adding complementary modules such as optical parametric oscillators (OPO) or optical parametric amplifiers (OPA), the $10 \mathrm{~nm}$ of spectral bandwidth can be tuned on a range between $500 \mathrm{~nm}$ and $2.5 \mu \mathrm{m}$. This allows much more fluorophores to be imaged. Moreover, three-photon probing processes can be involved: three-photon fluorescence (ThPF) and third harmonic generation (THG). But the resulting ability of imaging by TPF, SHG, ThPF and THG cannot be simultaneous. This drastically increases imaging delay, a critical parameter in the field of in vivo imaging or for detecting phenomena at video rates [7].

A huge quantity of custom-made excitation solutions for MPM can be found in literature and are summarized in [8, 9], most of them being monochromatic and femtosecond solutions. Considering the adaptation of continuous wave sources (CW) to MPM [10-13], the temporal aspect of laser pulses (pulse duration and repetition rate) is in fact not an incapacitating criterion for the generation of multiphoton probing processes contrary to what can be found in literature. MPM cannot be an alibi justifying the race for generating the shortest laser pulses. Reversely, reaching the electronic excitation state of the fluorophore involved in fluorescence phenomena is decisive in MPM. Indeed, multiphoton transition will never occur with a combined energy of excitation photons not adapted to the energy levels of the fluorophore, whatever the multiphoton process involved. Spectral bandwidth is therefore the exclusive physical parameter of excitation able to allow or prohibit multiphoton probing processes involved in MPM. Current developments in laser systems that can be found in literature address scarcely this key-point and keep the excitation wavelength fixed by the technology involved, thus presenting a restrained interest in MPM. In that context, a need in wideband excitation systems for MPM appeared, combined with specific detection solutions such as standard spectral detection or spatial autonomous real-time programmable fluorescence segmentation [14-16].

The compatibility of an ultra-wideband laser system (UWLS) with MPM has been highlighted about ten years ago [1723]. This solution consists usually in the spectral broadening of femtosecond laser pulses into a photonics crystal fiber (PCF) generating frequencies compatible with multiphoton excitation in the NIR. The wide spectrum can be shaped when coupled with sophisticated shaping modules such as liquid crystal matrix [24], spatial light modulator [12] or deformable mirror [25]. It allows simultaneous imaging of many fluorescent probes of interest in life sciences. But this technology is complex, expensive and not compatible with a turn-key solution, mandatory for a routine use. We have demonstrated the ability of a commercial nanosecond UWLS for MPM [26], illustrating the non-decisive aspect of temporal characteristics of the excitation laser pulses in MPM. Nanosecond UWLS are highly interesting thanks to its continuum of wavelength emitted by a relatively cheap and well-mastered physical principle. Very recently, the efficiency of a multicolor master oscillator power amplifier has also been demonstrated with three discreet wavelengths involved in MPM [27]. At that occasion, Eibl et al. have imparting the idea that a combination of long pulse duration with longer wavelengths could be ideal for deep tissue imaging. This original solution might however be considered keeping in mind that the longer excitation wavelengths are associated with a less favorable optical resolution [28]. Several computational solutions exist and constitute the main objective of new mathematical strategies [29-31].

In this publication, we propose a new method for multichannel multiphoton microscopy especially developed for imaging samples from life sciences, label-free and based on a wideband excitation spectrum. The interest of an ultrawideband laser excitation in MPM is first illustrated with the measurement of multiphoton absorption spectra of biological substances endogenously fluorescent. Then, we propose an original and simple laser setup, based on an UWLS coupled with a passive spectral shaping module suited to instantaneous multichannel MPM. Multiphoton images of samples from life sciences are presented, illustrating the adaptation of this system to MPM. Then, the UWLS and its shaping module are presented and characterized spectrally and temporally. 


\section{WIDEBAND ABSORPTION SPECTRA OF BIOLOGICAL SUBSTANCES ENDOGENOUSLY FLUORESCENT}

In biomedical researches, the validation of hypothesis goes through an imaging characterization and identification of specific probes. Thus, an infinity of biological targets require to be identified, located and quantified at microscopic scale. While most of them are not directly fluorescent and require a labelling process, several others have an endogenous fluorescence and are able to generate detectable light under an adapted excitation laser beam without any exogenous labeling molecule. Among these label-free fluorescent substances, the most know are proteins such as elastin, collagen or myosin, metabolic indicators such as NADH or FAD and single amino acids such as tryptophan, phenylalanine, glycine or tyrosine. Organic substances from plant origin are also good candidates for an endogenous fluorescence: cellulose, lignin or chlorophyll. In MPM, and especially in the case of endogenous fluorescence, the role of the spectral bandwidth of excitation is fundamental for allowing and optimizing a label-free absorption processes such as two-photon absorption (TPA). We notice here that a unique fluorophore can be excited thanks to a one-, two- or three-photon absorption process provided an adapted wavelength range of excitation. Figure 1 represents the experimental measurement of excitation spectra that we have measured from different substances of interest in life sciences.

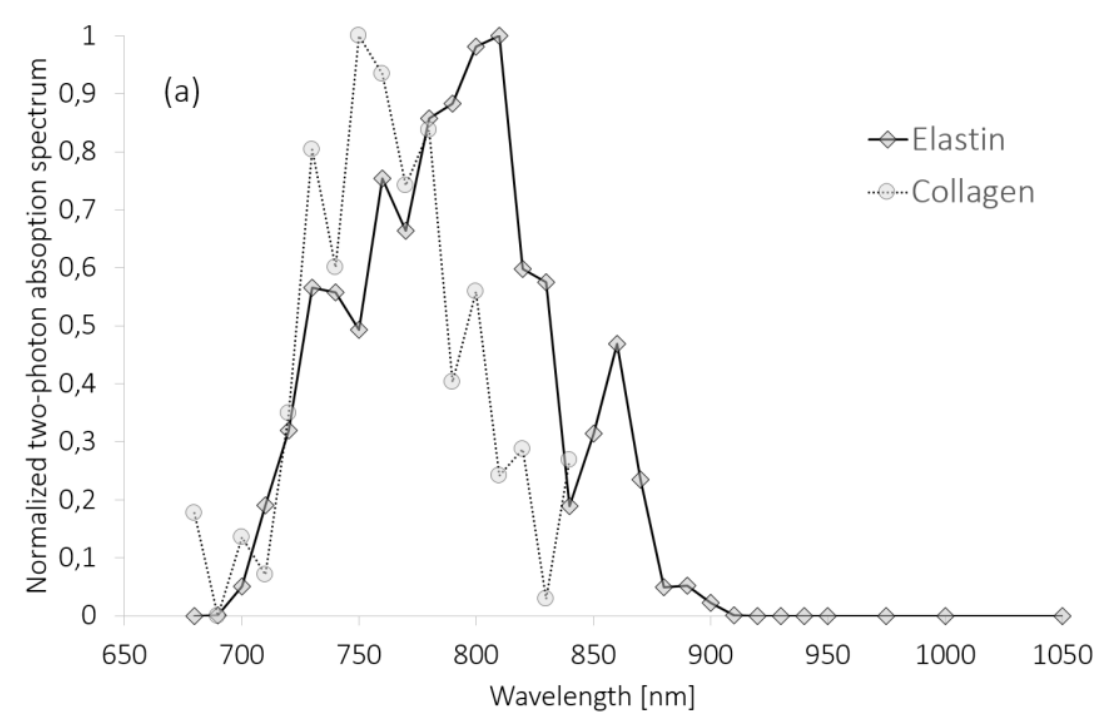

Figure 1. Experimental measurements of multiphoton excitation spectra of endogenous fluorescent biological substances of interest in life sciences. Measurements realized on synthetic and isolated samples, following Kao's protocol [32]. Two proteins: elastin and collagen; for collagen, the TPA spectrum is measured until $840 \mathrm{~nm}$. For longer excitation wavelengths, second harmonic generation is dominating the signal detected between 420 and $500 \mathrm{~nm}$.

For these two fluorescent substances usually present simultaneously in biological structures, their simultaneous imaging would require a continuous excitation spectrum from $700 \mathrm{~nm}$ until $900 \mathrm{~nm}$. Such an experimental protocol is not possible with a standard Ti: Sa source commonly implemented on multiphoton microscopes. Their simultaneous imaging imposes a time delay for switching the central wavelength of excitation. Despite the most recent technological and numerical advances for driving central wavelengths of tunable Ti: Sa sources, this time delay could never be reduced to zero. The simultaneous imaging of samples from life sciences by multispectral MPM requires a large spectral excitation bandwidth. The solution of an UWLS is thus optimal for generating instantaneous multichannel multiphoton imaging, a solution at the heart of next section. 


\section{MULTICHANNEL MPM WITH A LARGE BAND LASER SYSTEM}

\subsection{Experimental setup}

The objective of this section is to present our UWLS coupled with its shaping module implemented on a multiphoton microscope. The experimental setup is based on a standard configuration of upright multiphoton microscope stand from Thorlabs Inc. (Bergamo® II Series) where a scanning unit and a couple of photomultiplier tubes (PMTs) are connected to a computer for image display. Figure 2 summarized the overall optical assembly. Two laser sources can be alternatively coupled into the microscope stand. On the one hand, a standard Ti: Sa laser source (Chameleon Ultra II, Coherent Inc) is delivering pulses (150 fs), with a spectral bandwidth of $10 \mathrm{~nm}$ at the FWHM, spectrally tunable on the range $680-1050 \mathrm{~nm}$. On the other hand, a LBLS results from the spectral broadening into a microstructured fiber of a mode-locked laser, centered at $1064 \mathrm{~nm}$ and generating pulse durations of $5 \mathrm{ps}$ at $40 \mathrm{MHz}$. The spectral bandwidth available at fiber output covers a range between $410 \mathrm{~nm}$ until $2.4 \mu \mathrm{m}$ with a total average power of $2 \mathrm{~W}$, unequally distributed on the spectral range. At the fiber output, a parabolic mirror collimates the beam, before its injection into the spectral shaping module constituted by a prism-line which plays the role of spectral selection [33]. In this configuration, the prism-line plays the role of spectral shaping exclusively.

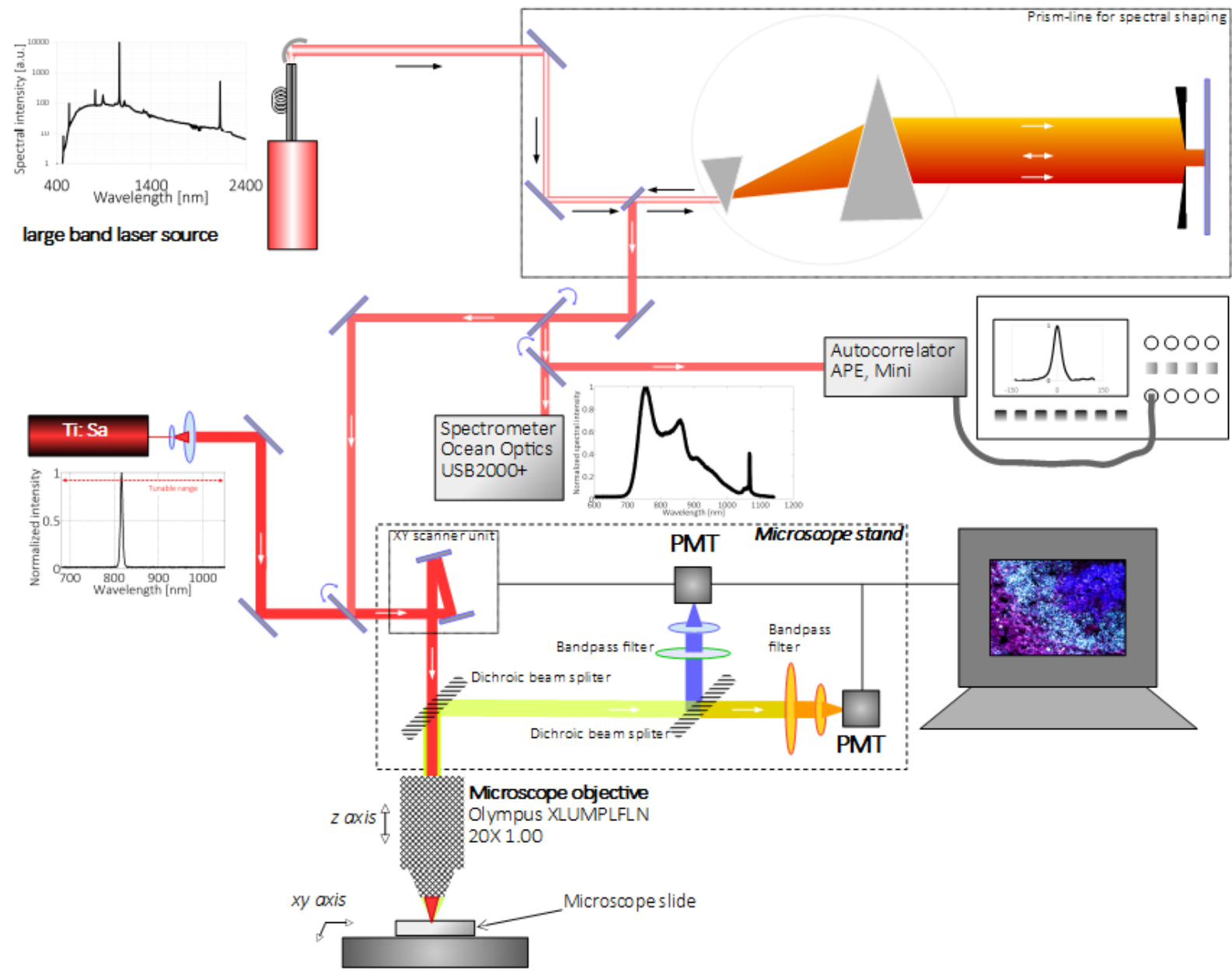

Figure 2. Experimental setup with a spectral shaping module of a LBLS for multiphoton imaging of samples from life sciences. 


\subsection{Illustration of multichannel multiphoton microscopy}

In the previous part, we have identified several biological compound endogenously fluorescent. Now we illustrate few of these optical properties particularly interesting for microscopic imaging. In that aim, a sample from the group of animals is used as an illustration. The spleen sample involving B Cells (Blue), T Cells (Cyan) and D cells (Magenta) are imaged (Figure 3A-C) and then merged (Figure 3D). Their TPF are detected respectively between $425 \mathrm{~nm}$ and $475 \mathrm{~nm}, 500 \mathrm{~nm}$ and $550 \mathrm{~nm}, 600$ and $650 \mathrm{~nm}$. The TPA spectrum of these four different fluorophores are spread between 680 and 1010 $\mathrm{nm}$. The excitation spectrum generated by our LBLS and its shaping module is centered at $900 \mathrm{~nm}$ with a spectral width of $330 \mathrm{~nm}$. Figure 3 illustrates the multiphoton images generated with this excitation beam.
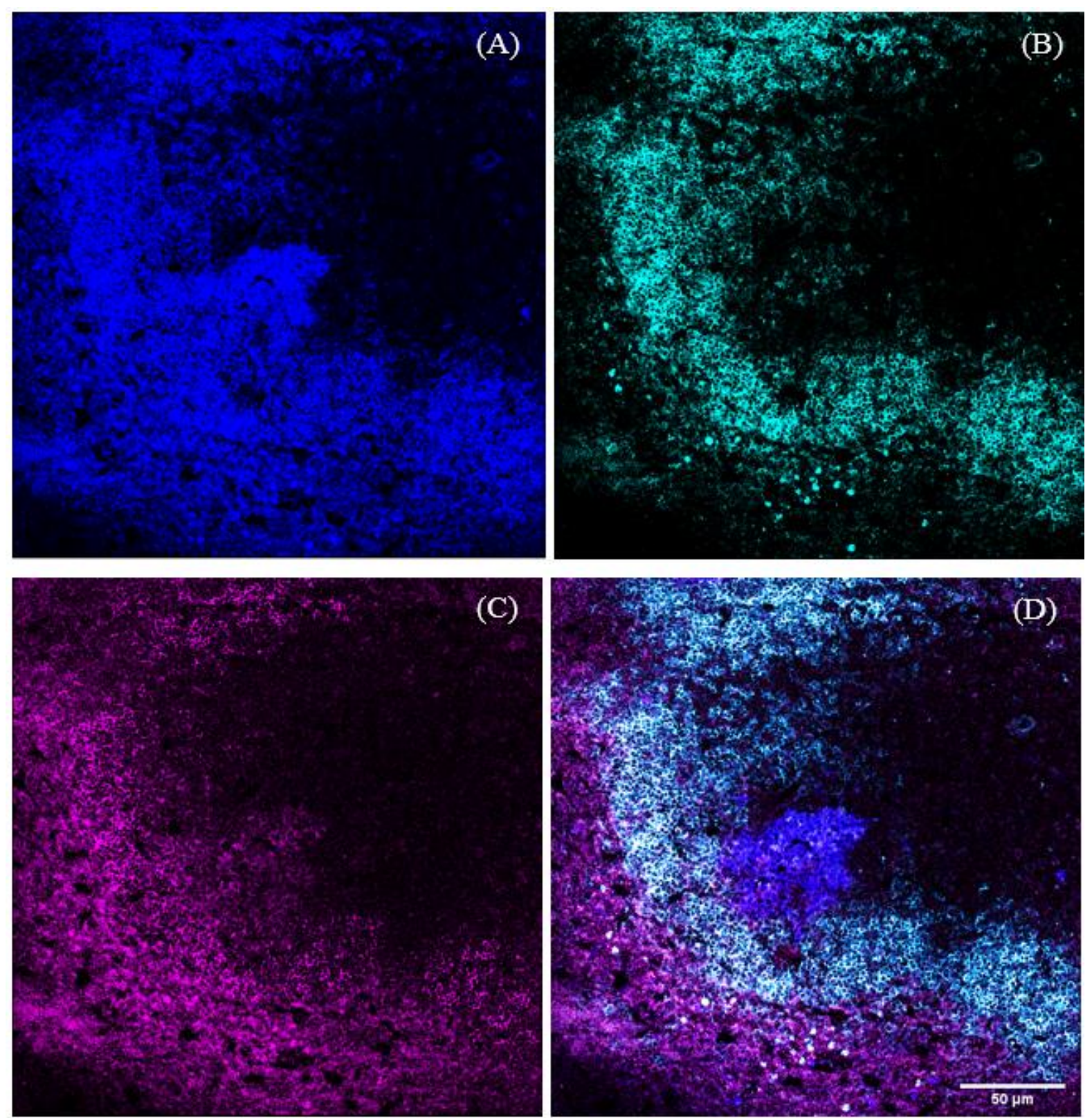

Figure 3. Illustration of multichannel multiphoton microscope with a large band laser system. Spleen images with three channels recorded. B Cells in Blue (A), T Cells in Cyan (B) and D cells in Magenta (C) are recorded with a unique excitation bandwidth. The three channels are then merged (D) for colocalizing the kind of cell constituting the spleen. 


\section{CONCLUSION}

The interest of a large band laser system spectrally shaped and coupled into a multiphoton microscope in order to image samples coming from life sciences by MPM at microscopic scale is experimentally illustrated in this presentation. The current way used here for generating large band laser pulses considers a spectral broadening of monochromatic short pulses from a microchip into a confined medium such as a photonic crystal fiber. Such a process is highly complex and is still the subject of many investigations in order to understand and master the physical phenomena involved. The physical phenomena occurring once the spectrum is established in free-space and shaped after the fiber output was not the goal of the present work. Here, we have shown that these picosecond laser pulses can be involved to generate multiphoton processes usually involved in MPM.

\section{REFERENCES}

[1] J. R. Lakowicz, "Principles of Fluorescence Spectroscopy", 3rd edn, Berlin: Springer (2006)

[2] A. Diaspro, "Confocal and Two-photon microscopy, Foundations, applications and advances", Wiley-Liss Inc., New York (2002)

[3] K. Radotic, A. Kalauzi, D. Djikanovic, M. Jeremic, R. M. Leblanc, Z. G. Cerovic, "Component analysis of the fluorescence spectra of a lignin model compound", Journal of Photochemistry and Photobiology B: Biology 83, $1-10(2006)$

[4] W. Denk, J. H. Strickler, W. W. Webb, “Two-Photon Laser Scanning Fluorescence Microscopy”, Science, 248 (4951), 73-76 (1990).

[5] P. T. So, C. Y. Dong, B. R. Masters, K. M. Berland, "Two-photon excitation fluorescence microscopy" Annual Review of Biomedical Engineering, 2, 399-429 (2000)

[6] B. R. Masters, P. T. So, "Handbook of Biomedical Nonlinear Optical Microscopy", Oxford University Press, 2008, 896 pages. ISBN 978-0-1951-6260-9

[7] N. Kirkpatrick, E. Chung, D. Cook, X. Han, G. Gruionu, S. Liao, L. Munn, T. Padera, D. Fukumura, R. K. Jain, "Video-rate resonant scanning multiphoton microscopy: An emerging technique for intravital imaging of the tumor microenvironment", IntraVital, 1 (1), 60-68 (2012)

[8] C. Lefort, "A review of biomedical multiphoton microscopy and its laser sources", Journal of Physics D: Applied Physics, 50, 423001 (2017)

[9] C. Lefort, "Laser sources in multiphoton microscopy: Overview and optimization", Proceedings of SPIE - The International Society for Optical Engineering, $106770 \mathrm{~V}$ (2018)

[10] E.L. Florin E L, J. K. Horber, E. H. K. Stelzer, "High-resolution axial and lateral position sensing using twophoton excitation of fluorophores by a continuous-wave Nd:YAG laser”, Applied Physics Letters, 69 (4), $446-$ 448 (1996)

[11] S. H. Hell, M. Booth, S. Wilms, C. M. Schnetter, A. K. Kirsch, D. J. Arndt-Jovin, T. M. Jovin, "Two-photon near and far-field fluorescence microscopy with continuous wave excitation", Optics Letters, 23 (15), 1238$1240(1998)$

[12] X. Hou, W. Cheng, "Single-molecule detection using continuous wave excitation of two-photon fluorescence", Optics Letters, 36 (16) 3185-3187 (2011)

[13]N. Uzunbajakava, C. Otto, "Combined Raman and continuous-wave-excited two-photon fluorescence cell imaging”, Optics Letters, 28 (21), 2073-2075 (2003)

[14]Z. X. Zhang, G. J. Sonek, X. B. Wei, C. Sun, M. W. Berns B. J. Tromberg, "Cell vialbility and DNA denaturation measurements by two-photon fluorescence excitation in CX Al:GaAs diode laser optical traps", Journal of Biomedical Optics, 4 (2), 256-259 (1999)

[15]K. Bacia, Z. Petrasek, P. Schwille, "Correcting for Spectral Cross-Talk in Dual-Color Fluorescence CrossCorrelation Spectroscopy", ChemPhysChem, 13, 1221 - 1231 (2012)

[16] J. Aluko, C. Perrin, V. Devauges, J. Nedbal, S. Poland, D. Matthews, J. Whittaker, S. Ameer-Beg, "Semiautonomous real-time programmable fluorescence lifetime segmentation with a digital micromirror device", Optics Express, 26 (24), 31055-31074 (2018) 
[17] J. A. Palero, V. O. Boer, J. C. Vijverberg, H. C. Gerritsen, "Short-wavelength two-photon excitation fluorescence microscopy of tryptophan with a photonic crystal fiber based light source", Optics Express, 13, 5363-5368 (2005)

[18] J. Tada, T. Kono, A. Suda, H. Mizuno, A. Miyawaki, K. Midorikawa and F. Kannari, “Adaptively controlled supercontinuum pulse from a microstructure fiber for two-photon excited fluorescence microscopy” Appl. Opt. 46 3023-30 (2007)

[19]D. Li, W. Zheng, J. Y. Qu, “Two-photon autofluorescence microscopy of multicolor excitation”, Optics Letters, 34 (2), 202-204 (2009)

[20] Tao W, Bao H and $\mathrm{Gu}$ M 2011 "Enhanced two-channel nonlinear imaging by a highly polarized supercontinuum light source generated from a nonlinear photonic crystal fiber with two zero-dispersion wavelengths", J. Biomed. Opt., 16, 056010 (2011)

[21] H. Tu, Y. Liu, D. Turchinovich, M. Marjanovic, J. Lyngs, J. Lægsgaard, E. J. Chaney, Y. Zhao, S. You, W. L. Wilson, B. Xu, M. Dantus, S. A. Boppart, "Stain-free histopathology by programmable supercontinuum pulses" Nature Photonics, 10 (8), 534-540 (2016)

[22] Q. Cui, Z. Chen, Q. Liu, Z. Zhang, Q. Luo, L. Fu, "Visible continuum pulses based on enhanced dispersive wave generation for endogenous fluorescence imaging”, Biomedical Optics Express, 8 (9), 4026 - 4036 (2017)

[23] C. Poudel, C. F. Kaminski, "Supercontinuum radiation in fluorescence microscopy and biomedical imaging applications", JOSA B, 36 (2), A139-A153 (2019)

[24] C. Lefort, M. Kalashyan, G. Ducourthial, T. Mansuryan, R. P. O'Connor, F. Louradour, "Sub-30 fs pulse compression and pulse shaping at the output of a 2 m-long optical fiber in the near-infrared range", JOSA B, 31, 2317-24 (2014)

[25] O. Albert, L. Sherman, G. Mourou, T. B. Norris, G. Vdovin, "Smart microscope: an adaptive optics learning system for aberration correction in multiphoton confocal microscopy", Optics Letters, 25 (1), 52-54 (2000)

[26] C. Lefort, R. P. O’Connor, V. Blanquet, L. Magnol, H. Kano, V. Tombelaine, P. Lévêque, V. Couderc, P. Leproux, "Multicolor multiphoton microscopy based on a nanosecond supercontinuum laser source" J. Biophotonics, 9, 709-14 (2016)

[27] M. Eibl, D. Weng, H. Hakert, J. P. Kolb, T. Pfeiffer, J. E. Hundt, R. Huber, S. Karpf, "Wavelength agile multiphoton microscopy with a fiber amplified diode laser”, Biomedical Optics Express, 9 (12), 6273-6282 (2018)

[28] J. Mertz, "Introduction to Optical Microscopy", Roberts \& Company Publishers (2009)

[29]T. K. Lau, E. Chouzenoux, C. Lefort and J.-C. Pesquet, "Optimal Multivariate Gaussian Fitting for PSF Modeling in Two-Photon Microscopy", Proceedings of the IEEE International Symposium on Biomedical Imaging (2018)

[30]E. Chouzenoux, T. Tsz-Kit Lau, C. Lefort, J.-C. Pesquet, "Optimal Multivariate Gaussian Fitting with Applications to PSF Modeling in Two-Photon Microscopy Imaging”, Journal of Mathematical Imaging and Vision, 61, 7, 1037-1050 (2019)

[31] Robert Gonsalves, "Phase diversity: Math, methods and prospects, including sequential diversity imaging" Proceedings of SPIE - The International Society for Optical Engineering, 10677 (2018)

[32]F.-J. Kao, "The Use of Optical Parametric Oscillator for Harmonic Generation and Two-Photon UV Fluorescence Microscopy", Microscopy Research and Technique, 63, 175-181 (2004)

[33] C. Dunsby, P. M. P. Lanigan, J. Mc Ginty, D. S. Elson, J. Requejo-Isidro, I. Munro, N. Galletly, F. Mc Cann, B. Treanor, B. Onfelt, D. M. Davis, M. A. A. Neil, P. M. W. French, "An electronically tunable ultrafast laser source applied to fluorescence imaging and fluorescence lifetime imaging microscopy", Journal of Physics D: Applied Physics, 37 3296-3303 (2004) 